\title{
Féeries
}

Études sur le conte merveilleux, XVII $-\mathrm{XIXe}$ siècle

\section{Ce que les contes doivent aux fées. Liaisons anthropologiques}

What Fairy Tales owe to Fairies: Anthropological Connections

Jean-Paul Sermain

\section{(2) OpenEdition}

1 Journals

Édition électronique

URL : http://journals.openedition.org/feeries/768

DOI : $10.4000 /$ feeries.768

ISSN : 1957-7753

Éditeur

UGA Éditions/Université Grenoble Alpes

\section{Édition imprimée}

Date de publication : 31 octobre 2010

Pagination : 193-201

ISBN : 978-2-84310-182-3

ISSN : 1766-2842

\section{Référence électronique}

Jean-Paul Sermain, «Ce que les contes doivent aux fées. Liaisons anthropologiques », Féeries [En ligne], 7 | 2010, mis en ligne le 31 juillet 2011, consulté le 08 septembre 2020. URL : http:// journals.openedition.org/feeries/768; DOI : https://doi.org/10.4000/feeries.768

\section{(c) Féeries}




\title{
CE QUE LES CONTES DOIVENT AUX FÉES. LIAISONS ANTHROPOLOGIQUES
}

\author{
À Aurélia Gaillard
}

$\Gamma$

'EXAMEN DU CONTE AU REGARD DE LA FABLE peut être poussé plus avant, en distinguant le conte de fées du conte. Dans la confrontation du conte de fées au conte et à la fable, l'originalité de son propos se détachera par contraste; on verra aussi ce qu'il importe des traits plus généraux du conte et de la fable et ce qu'il en fait : quelles ressources de ces deux genres exploite-t-il et transforme-t-il? Le terme générique de "conte de fées» (trouvé et fixé très tôt, en I698, et qui fait défaut à de nombreuses langues et littératures) le caractérise par son contenu, par un type de personnages venus du Moyen Âge, possédant des pouvoirs magiques et perçus, dans ce genre nouveau, comme purement fantaisistes et imaginaires (à l'instar des animaux parlant de la fable). Raymonde Robert a donc obéi à cette indication et défini le genre par ses fées et par la scène des dons qu'elles apportent ${ }^{1}$. De façon plus générale, l'intervention des fées introduit dans l'histoire une dimension surnaturelle qui en affecte profondément l'économie, mais leurs propriétés floues, leur place restreinte (les personnages principaux sont naturels) et surtout leur statut très vague ne permettent pas de conférer à cette dimension surnaturelle la moindre consistance : les fées ne s'intègrent à aucune croyance, ne renvoient à aucune légende, ne possèdent aucun pedigree. Quasiment sans lieu et sans domaine. Ce renvoi très ténu à un ailleurs improbable, à un surnaturel presque aussi emprunté que celui des opéras, est pourtant décisif, et c'est par le biais des liaisons avec le conte et la fable qu'on peut parvenir à en capter l'enjeu. Il suffit pour cela de se laisser guider par les trois acceptions que l'époque donne au mot fable.

I. R. Robert, Le Conte de fées littéraire en France de la fin du XVII siècle à la fin du XVIII siècle, Paris, Champion, 2002 [Presses Universitaires de Nancy, I982], p. 5I-82. 
La première est celle qui aujourd'hui vient la première à l'esprit, et que nous associons à La Fontaine. Nous avons vu que la fable entre dans des projets rhétoriques et se définit de façon plus large par des dispositifs herméneutiques. Ainsi la fable du loup et de l'agneau s'organise comme un procès qui oppose les deux partis. La moralité initiale tire sa conclusion de l'issue du procès : «La raison du plus fort est toujours la meilleure.» Elle oppose la force à la justice et l'évaluation «meilleure» réunit les deux principes au prix d'une contradiction ou d'un sophisme : ce qui est tenu pour «meilleur» et s'impose au droit ne l'est que par la force. Le «meilleur» ne vaut que pour l'appétit du loup, mais nullement dans le champ, légal et civil, du procès. Les trois termes (raison, fort, meilleure) introduisent et articulent trois registres de valeurs fondamentaux dans toute société humaine et les confrontent au terme final de la fable, la satisfaction de l'appétit du loup, la dévoration de l'agneau. S'oppose ainsi à un ordre (celui du procès) une violence proprement brutale : le loup est une figuration de l'excès, d'un passage au-delà des limites de l'humain. La convention du personnage animal et parlant est mise au service d'une interrogation morale et politique : un ordre est-il possible avec des hommes loups? La «féerie» du conte va fournir un moyen analogue mais sans la médiation de la scène rhétorique, en retrouvant l'implication de la double nature humaine et animale dans l'ouverture du monde familier aux pouvoirs de la fée.

Il faut se tourner vers le discours critique de la Poétique pour trouver un autre emploi de fable. On l'a vu, fables et contes se définissent tous deux par le traitement du récit, la clarté de son parcours et le sens de son achèvement. Une telle qualification de l'histoire est alors appréhendée par le mot "fable", qui sert en effet à traduire le terme qu'Aristote appelle "muthos» et qu'il introduit dans l'énumération des "parties» qui font la tragédie : "Il y a donc nécessairement dans toute Tragédie six choses : la Fable, les Mours, les Paroles, les Pensées, le Spectacle, le Chant.» Précédemment: "J'appelle Fable l'arrangement des parties dont est composée une action poétique ${ }^{2}$.» Il explique ensuite comment une fable est "une» et "entière» :

Il faut que la fable soit l'imitation d'une seule action; que cette action soit entière; et que les parties soient tellement liées entre elles, qu'une seule transposée ou retranchée, ce ne soit plus un tout, ou le même tout. Car tout ce qui peut être dans un tout, ou n'y être pas, sans qu'il y paraisse, n'est point partie de ce tout ${ }^{3}$.

2. Traduction due à l'abbé Batteux dans Les Quatre Poétiques, Paris, Saillant \& Nyon, I77I, p. 59.

3. Ibid., p. 79 . 
Aristote a fait précéder cette règle d'une réflexion sur l'étendue de la fable prenant en compte les conditions de sa perception :

Si l'on considère la nature même de la chose, plus une pièce aura d'étendue, plus elle sera belle. En un mot, elle aura l'étendue qui lui sera nécessaire, pour que les incidents, naissant les uns des autres, nécessairement ou vraisemblablement, amènent la révolution du bonheur au malheur ou du malheur au bonheur.

Le récit court, fable, conte ou nouvelle, répond au mieux à cet idéal défini pour la tragédie (beaucoup mieux que le roman et même l'épopée homérique) : la saisie d'une vision d'ensemble rendue par l'enchaînement limité des épisodes joue un rôle majeur dans la transmission du conte, et c'est ce qu'ont bien compris a contrario tous ceux qui veulent perturber cette saisie cavalière, les parodistes comme Hamilton (multipliant déboîtements et épisodes), ou les originales comme $\mathrm{M}^{\mathrm{lle}}$ de Lubert : elle s'amuse à détruire le conte (aussi ne peut-on mémoriser les siens : ils valent au mieux par leur flux éphémère, comme un film d'Andy Warhol).

La transparence du conte à sa «fable», au sens de «muthos» d'Aristote, lui permet deux développements que le conte de fées va s'approprier, l'un et l'autre engagent le temps et la conscience du temps. Ils sont corrélés. Le conte, plus que tout autre genre narratif, fable, nouvelle, roman, épopée par exemple, met tous ses éléments au service de l'histoire, de la clarté des enchaînements et de la nécessité du terme qui les conclut. Ainsi dans le conte assez négligé de Perrault, Les Fées, chacune des deux sœurs agit selon les qualités qui lui sont échues dans la famille, et celles-ci ne valent que dans une opposition qui structure leur histoire et conduit l'une à la cour et au trône, et l'autre à une mort de brute abandonnée dans la forêt. Chaque trait des héros est ainsi subordonné à l'histoire qui les utilise et les domine et qui possède sa propre finalité : d'être précisément une histoire, qu'on retient, qu'on échange, qu'on transmet, qu'on transforme. Le conte, qui écarte la liberté des personnages (caractéristique de la nouvelle) et leur position rhétorique (propre à la fable), se laisse tout entier ramener à l'aspect perfectif du récit, à son achèvement. Le conte, par sa forme narrative, peut susciter le sentiment heureux d'un accomplissement - tout conduit à bonne fin - ou d'une inexorable machinerie — le héros n'est que le jouet d'un ordre supérieur. La place qu'Aristote donne à la fable dans la tragédie témoigne de la virtualité tragique de tout récit, quand tout se lit à partir d'une fin implacable. André Jolles a souligné le lien du conte de fées (exactement le Märchen) au tragique, posé et suspendu selon lui ${ }^{4}$. L'aprèscoup et le trop tard. Le conte donne une densité à ce qui s'écoule dans le

4. A. Jolles, Formes simples [Einfache Formen], Paris, Éditions du Seuil, 1972 [1930], p. 173-195. 
temps, modalise l'expérience et garantit sa communication, il retient la nuit, mais sur un mode où elle est déjà passée. On garde du conte le sentiment du révolu. La forme du conte et les effets qu’elle produit ménagent ainsi son autre articulation dans le temps qui est essentielle pour le conte de fées : le conte fixe la mémoire et assure la transmission des histoires, aussi se forme-t-il au sein d'une communauté, dans des échanges et des appropriations, il passe d'une génération à l'autre et se colore de cette filiation et de ces continuités. Il rend sensible la tradition qu'il forge luimême : toujours prêt à actualiser le passé et à le relier au présent.

Le mot «fable» a une troisième acception qu'on pourrait considérer comme une spécialisation de la seconde (comme le conte de fées est une spécialisation du conte). Elle est signalée dans l'usage de la première modernité en français par une majuscule. La Fable, c'est «l'histoire des Dieux et des Héros fabuleux de l'antiquité, et l'explication des mystères de leur fausse religion, de leurs fables et de leurs métamorphoses", histoire qui porte alors le nom de "mythologie`». Les récits de cette "histoire mythologique» se distinguent de ceux des trois grandes religions révélées — juive, chrétienne, mahométane - par leur fausseté évidente, mais aussi sur un autre plan. Ceux-ci sont historiques, renvoient à des événements connus et $\mathrm{y}$ intègrent les interventions divines, au point même que la naissance du Christ sert de repère chronologique presque universel et date l'ensemble des événements. Les récits de la Fable sont, eux, sans accroche historique, ils sont multiples et ont des intrigues presque indépendantes avec des acteurs différents (certes souvent récurrents). Ces récits s'apparentent aux contes par leur mode d'échange, de transmission et de transformation, et par leurs contours fermes, leurs motivations élémentaires, leurs conclusions inexorables, de goût souvent tragique. Les Métamorphoses d'Ovide font ainsi le pont entre la Fable et le recueil de contes merveilleux, on peut même considérer que c'est le premier et le principal d'entre eux, puisant aux seules sources de la Fable. Le chef-d'œuvre latin, constamment retraduit et réédité aux $\mathrm{XVII}^{\mathrm{e}}$ et XVIII ${ }^{\mathrm{e}}$ siècles, révélait et illustrait l'articulation du dispositif littéraire du conte avec une disposition anthropologique qu'il est difficile de saisir parce que la première modernité se l'est en partie appropriée sans trop s'expliquer (sauf peut-être bien tardivement dans la Scienza Nuova de Vico) et que la seconde modernité l'a développée, étendue et assimilée sous le nom de «mythe»: récit d'une origine, d'un temps originaire, où l'univers humain est perméable à l'intervention des

5. Dictionnaire de Trévoux, édition de $\mathbf{1 7 7 7 .}$ 
dieux et des forces surnaturelles, c'est-à-dire où les histoires et les passions humaines ne peuvent être racontées qu'en intégrant une sorte d'expansion magique et religieuse, où l'ordre humain doit faire place à des valeurs et à des causalités qui le débordent mais aussi le déterminent et lui donnent un sens.

On peut heureusement s'appuyer sur l'ouvrage magistral d'Aurélia Gaillard $^{6}$, qui aborde le conte de fées dans la perspective historique d'une transformation, dans la seconde moitié du XVII ${ }^{\text {e }}$ siècle, du rapport à la Fable, de son usage et de sa perception. Selon cette sorte de reconstitution archéologique, la Fable consiste à distinguer et à articuler deux temps, celui du fabuleux, donc étranger à toute mesure, qui habite l'histoire racontée et celui, ancré, lui, dans l'actualité et la chronologie du récit qui en est fait. La Fable implique donc la prise de conscience d'un rapport à un autre temps et à un autre ordre qui concerne l'ensemble des composantes de l'histoire, les personnages, les enchaînements, les motifs, les causes, etc. À la fin du XviI ${ }^{\text {e }}$ siècle, ce contact médiat avec le fabuleux se perd, se défait, et la Fable devient un répertoire de déguisements, souvent hédonistes.

Le conte de fées français, historiquement, opère une sorte de transfert de ce double dispositif littéraire et anthropologique de la Fable au sein de la culture chrétienne et de son propre passé national. Il retient de la Fable deux traits au moins : l'opposition au présent (actualisé à la fois dans son intégration allusive à un loisir galant et mondain et dans l'évidence du livre, la communication imprimée, ouverte et indéterminée) d'un temps second que les premiers auteurs de contes caractérisent par une scène de formation et de transmission de l'histoire à la fois chrétienne, pédagogique et paysanne, mais comme rendue anonyme dans la succession toujours identique des générations. Le conte de fées se présente, nous dit A. Gaillard, comme la répétition d'un texte antérieur. Cette seconde temporalité ne s'oppose pas seulement au présent par sa distance (marquée par des archaïsmes de tous ordres) mais, comme la Fable, par son ordre : échappant à toute mesure et à toute chronologie. La translation de la Fable dans le conte passe par la reprise de son dispositif et de ce qu'on pourrait appeler une même disposition anthropologique dans la modélisation de l'expérience : ou du moins le conte de fées amène rétrospectivement, s'appuyant sur elle, à lire de façon analogue à ce qu'il met en place les récits de la Fable que nous versons au chapitre des «mythes». Le conte

6. A. Gaillard, Fables, mythes, contes - l'esthétique du fabuleux (I660-I724), Paris, Champion, 1996. 
de fées exploite et transpose cette distance à l'égard d'un passé «fabuleux", s'en sert pour introduire la trace d'une surnature dans la perception des conduites (en particulier amoureuses), des violences, des transgressions des lois humaines et civiles : la Fable et le conte de fées confrontent l'homme à ce qui échappe à son ordre et à sa maîtrise (d'où sa reprise par la psychanalyse). Le conte recourt à de telles histoires fabuleuses comme à un mode de pensée imaginatif et ludique : en cela il fonctionne exactement comme la future nouvelle fantastique.

Le conte de fées obligeait ses auteurs à marcher sur des œufs. D'une part, ils devaient loger dans la culture et la mémoire française (ou dans son imaginaire arabe pour Galland) un monde conforme à la disposition anthropologique de la Fable, fait d'allusions, de bribes de merveilleux, d'histoires anciennes, d'éléments archä̈ques et populaires, enfantins et maternels. Ils devaient en outre se servir de ce «monde des fées", puisque c'est sa dénomination, pour saisir les aventures humaines dans une dimension transcendantale, dans une sorte de débordement de forces obscures ou d'aspirations à une ascension exaltante. On a toujours su que trop de contes sont vraiment ennuyeux et immédiatement oubliables, parce qu'ils manquent cette raison d'être du fabuleux et s'en servent au mieux pour brocher sans fin des histoires trop faciles. La distance temporelle permettait, au contraire, de placer les personnages à la fois dans la logique du présent (qui était alors celle de la "nouvelle», dont se rapprochent plusieurs contes) et dans celle du fabuleux, c'est-à-dire dans l'illimité, quand les tendances, les désirs et les volontés s'affranchissent de toute limite, font ainsi perdre aux personnages leur place morale et civile, humaine et prosaïque : les voilà transportés. Peu de contes ont su répondre à cet appel du fabuleux. Perrault l'a entendu, de Villeneuve et Leprince de Beaumont dans le conte qui porte le même titre de La Belle et la Bête, d'Aulnoy parfois; Galland quand il a découvert le manuscrit des Mille et Une Nuits. Leurs contes sont ouverts à ce qui dans l'homme vient l'arracher à son univers familier et l'emporter au-delà de lui-même dans un mouvement d'ascension ou dans un tourbillon de violences.

L'exaltation de l'amour en faisait, de Dante à Pétrarque, une voie d'accès à la surnature et même à Dieu. Perrault en garde quelque chose dans deux contes, La Belle au bois dormant et Cendrillon. Cette exaltation n'est plus rapportée à l'adoration masculine. Elle est réalisée au sein même de l'amour et affecte la femme de façon privilégiée, elle s'incarne en elle en épousant le mouvement fabuleux de la métamorphose. Dans les deux cas, l'ascension ne part pas d'une approche sensuelle de l'amour mais d'une persécution antinaturelle qui, pour la deuxième partie de La Belle au bois 
dormant, tient même au cannibalisme. Le récit cadre des Mille et Une Nuits, qui contient à la fois des fables merveilleuses et un épisode d'érotisme magique (la femme enfermée par le génie transforme le commerce charnel en oppression violente), suggère aussi la dimension surnaturelle du lien entre les deux époux - l'association du mariage et du meurtre, de la loi et de sa destruction, étant poussée à son point extrême, tout comme le renversement opéré par le trésor des contes ouvert par Scheherazade, capable de restaurer un État, un homme et un mariage : de refonder tout l'ordre humain. De nombreux contes sont portés par la même dynamique, mais en lui donnant un parcours moins tranché, en l'enrichissant de variations épisodiques et donc en développant la fable au sens $\mathrm{du}$ "muthos» d'Aristote $^{7}$. L' «Histoire des amours de Caramalzaman, prince de l'île des Enfants de Khaledan et de Badoure, princesse de la Chine», aussi bien que la plus connue "Histoire de trois Calenders, fils de roi, et de cinq dames de Bagdad", si aimée de Walter Scott (qui laisse son narrateur de The Heart of Midlothian s'y référer plusieurs fois), réunissent avec éclat et élégance les différentes composantes du conte fabuleux : l'emportement des deux pères empêche la réalisation aisée de leur projet de marier leurs futurs deux enfants et amène un circuit narratif "arabesque», les accidents successifs délivrent les époux de l'emprise familiale et sociale au profit, d'une part, de l'amour (amour à la fois immédiat, hasardé et charnel des deux jeunes gens mais aussi amour du fils pour la mère comme pour son propre fils), au profit, d'autre part, de la fidélité par-delà les déserts et les années - et tout cela grâce aux génies et aux fées qui, curieusement, ignorent à quelle œuvre ils participent mais répondent à l'appel d'une beauté transcendante et à un ordre idéal des valeurs (où le beau ne peut être soumis au laid, le pauvre au pouvoir). L'exaltation amoureuse naît d'une rencontre des corps et celle-ci apparaît à la fois comme un acte de justice civile et le résultat d'interventions surnaturelles. Le tracé de la fable dessine donc une ascension amoureuse, un accomplissement familial et un parcours magique : la réussite du conte vient de la fusion des trois éléments.

Plusieurs contes de Perrault choisissent plutôt de figurer les forces obscures qui traînent l'homme dans l'horreur et le néant — infra humanité de la faim où chacun peut devenir un loup pour l'autre, dans une menace générale d'anthropophagie, ce que Le Petit Poucet raconte sur un registre sombre et Le Chat botté sur un registre gai : ici la menace vient de la société qui contient en elle sa propre destruction, figurée dans les deux contes

7. Voir J. E. Bencheikh, Les Mille et Une Nuits ou la Parole prisonnière, Paris, Gallimard, I988. 
de Perrault par les ogres venant relayer la volonté meurtrière des parents (les deux étant liés par la même faim). Dans les deux cas, cet abaissement sans fond conduit le héros à trouver en lui l'énergie extraordinaire (mais humaine) qui lui permettra de remonter et d'accéder à la réussite, d'une certaine façon aussi merveilleuse que l'anéantissement programmé. De nombreux contes des Mille et Une Nuits, les plus proches du "folklore", donnent au fabuleux une place assez voisine dans l'itinéraire du héros, soit que celui-ci s'oppose à des forces naturelles extraordinaires sous la forme de monstres (ainsi pour Sindbad), ou que celui-ci connaisse alternativement des magiciens hostiles et bienveillants comme Aladdin, dont le destin s'accomplit aussi dans l'exaltation merveilleuse de l'amour (sur un mode d'enchaînement, du trivial au magique, assez voisin des autres contes des Nuits).

C'est surtout dans le champ amoureux que l'individu découvre en lui une puissance qui peut le déborder et détruire le partenaire : la menace sur l'intégrité de l'être n'est plus liée à des conditions sociales mais à une nature intérieure, celle du désir qui ravit le Petit Chaperon Rouge, celle de Barbe bleue accumulant les meurtres de ses épouses successives. La Belle et la Bête (dont de nombreuses conteuses ont donné d'excellentes versions mais restées aux portes de la mémoire collective) reprend cette situation et combine de façon heureuse la menace que représente l'animalité brutale dans l'amour, quelque chose de destructeur pour les deux amants (ainsi que le dit d'Aulnoy dans Le Prince Marcassin), soumis à une dégradation égale, avec une valeur d'épreuve que prend cette expérience, permettant aux personnages de se dépasser et de connaître la générosité de l'amour.

Le conte de fées ainsi se nourrit de la Fable et en propose une version moderne capable en retour d'assurer la lecture de ce qu'elle transpose. Cette relation n'a pu apparaître que progressivement et alors que le vocable de Fable disparaissait au profit de celui de mythe qui, au moins dans son original grec, se confondait avec le principe de l'histoire. Fable et fable, muthos et mythes, leur croisement n'a pu se faire que rétrospectivement, en s'appuyant sur des notions anciennes, mais n'ayant pas développé leurs ressources sémantiques, ni leurs liaisons. Elles permettent de reconstituer une anthropologie poétique idéalement réalisée dans peu de contes. Faut-il pour autant réduire la portée mythique de ces contes à leur audience et à leur aura : est-elle ce que le public en fait? Oui, dans le sens, on l'a dit, où le conte se définit par l'histoire même de sa transmission. Mais sans doute faut-il regarder aussi vers les usages modernes du conte, c'est-à-dire vers l'enfance et la jeunesse, et vers la tentative la plus réussie 
pour retrouver l'esprit du fabuleux dans le titre même choisi par Bruno Bettelheim, The Uses of enchantment ${ }^{8}$.

Si l'"Histoire d'Ali Baba et de quarante voleurs exterminés par une esclave" prend, de tous les contes des Nuits, une valeur mythique, c'est en partie par ses transformations populaires et pour la jeunesse, mais aussi peut-être à cause du visage et du sens qu'y prend le fabuleux : la dimension magique y est retreinte, ludique (dans le recours à une formule avec les épisodes qui y sont liés) et surtout elle incarne l'ambivalence du surnaturel puisqu'elle ouvre à une richesse de rêve comme elle appartient à une organisation criminelle en marge de l'ordre social et venant le miner sourdement. L'enterrement du frère coupé en quatre quartiers prolonge cette ambivalence car cet acte de conformation à la civilité religieuse à la fois s'inscrit dans le conflit qui structure la fable (le «muthos»), témoigne de l'ingéniosité des héros et passe par une sorte de "couture» merveilleuse, de remembrement miraculeux. L'épisode annoncé dans le titre et qui clôt le conte fait se rencontrer la violence déchaînée du chef des voleurs et l'astuce suprême de l'esclave : ici l'extraordinaire vient donner à ces deux mouvements antagonistes de la brutalité et de l'ascension leur dimension surnaturelle. Le fabuleux se confond avec l'esprit du conte (celui que porte le tracé de l'histoire), son caractère magique est assez limité et plaisant, et il se prolonge dans l'action surhumaine des héros, dans ce qui fait leur grandeur, et cette grandeur est toute entière incarnée dans une prose humble et familière, humoristique, avec une sorte de distance dans l'émotion qu'implique ce terme.

8. B. Bettelheim, The Uses of Enchantment: the Meaning and Importance of Fairy Tales, New York, Vintage books, 1977. 